\title{
Estudio clínico controlado para valorar la utilidad del ancho de distribución plaquetaria (PDW) en el diagnóstico de apendicitis aguda
}

\author{
Controlled clinical study to evaluate the utility of the platelet distribution width (PDW) in \\ the diagnosis of acute appendicitis
}

\author{
Rafael A. Espinosa-Campos ${ }^{1,2 *}$, Silvia G. De la O-Hernández ${ }^{3}$, María G. González-Zárate ${ }^{3}$ y \\ Eduardo E. Montalvo-Javéz,4 \\ ${ }^{1}$ División de Cirugía, Unidad Médica de Alta Especialidad No. 71, Instituto Mexicano del Seguro Social (IMSS), Torreón Coahuila; ${ }^{2}$ Departamento \\ de Cirugía, Facultad de Medicina, Universidad Nacional Autónoma de México, Ciudad de México; ${ }^{3}$ Servicio de Cirugía General, Hospital General \\ Dr. Fernando Quiroz Gutiérrez, Instituto de Seguridad y Servicios Sociales de los Trabajadores del Estado (ISSSTE), Ciudad de México; ${ }^{4}$ Servicio \\ de Cirugía General, Clínica de Cirugía Hepato-Pancreato-Biliar, Hospital General de México Dr. Eduardo Liceaga, Ciudad de México. México
}

\section{Resumen}

Antecedentes: La apendicitis aguda es la urgencia abdominal más común en el mundo. Estudios recientes han investigado la eficacia del ancho de distribución plaquetaria (PDW, platelet distribution width) en el diagnóstico de apendicitis aguda. Objetivos: Determinar si existe correlación entre el PDW y el diagnóstico de pacientes con apendicitis aguda, y definir su relación respecto a las fases de apendicitis. Método: Se realizó un estudio de casos y controles con dos grupos poblacionales, conformados por 41 pacientes mayores de 18 años con diagnóstico de apendicitis aguda (grupo l) y 41 sujetos sanos (grupo II). El análisis estadístico se realizó utilizando el programa SPSS versión 21. Resultados: Se observó una edad media de $37.27 \pm 15.6$ años. La mayor cantidad de casos de apendicitis se presentaron en la fase III. La cifra total de leucocitos y el porcentaje de neutrófilos mostraron diferencias estadísticamente significativas. Se observó que no existe asociación entre la fase de apendicitis y los valores de PDW. Conclusiones: No hay diferencias entre los valores de PDW de pacientes con apendicitis aguda en comparación con sujetos sanos, ni relación del PDW con las fases de la apendicitis.

Palabras clave: Apendicitis aguda. Diagnóstico. PDW.

\begin{abstract}
Introduction: Acute appendicitis is the most common abdominal urgency around the world. Recent studies have investigated the efficacy of platelet distribution width (PDW) in the diagnosis of acute appendicitis. Objectives: To determine if there is a correlation between PDW and the diagnosis of patients with acute appendicitis and to define their relation to the phases of appendicitis. Method: A case-controlled study was conducted in two population groups with 41 patients older than 18 years of age diagnosed with acute appendicitis (group I) and 41 healthy patients (group II). Statistical analysis was performed using SPSS software version 21. Results: An average age of $37.27 \pm 15.6$ years was observed. The largest number of cases with acute appendicitis were presented in phase III. The total number of leukocytes and the percentage of neutrophils presented significant differences in the statistics. It was observed that there is no association between the appendicitis phase and the
\end{abstract}

\section{Correspondencia:}

*Rafael A. Espinosa-Campos

Dr. Balmis, 148

Col. Doctores, Del. Cuauhtémoc

Fecha de recepción: 13-12-2018

C.P. 06720, Ciudad de México, México

E-mail: alejandro.escam@gmail.com 0009-7411/๑ 2020 Academia Mexicana de Cirugía. Publicado por Permanyer. Este es un artículo open access bajo la licencia CC BY-NC-ND (http://creativecommons.org/licenses/by-nc-nd/4.0/).

Cir Cir. 2020;88(5):630-634 Contents available at PubMed www.cirugiaycirujanos.com 
PDW values. Conclusion: There are no differences between PDW values of patients with acute appendicitis compared to healthy patients, nor PDW ratio with the phases of appendicitis.

Key words: Acute appendicitis. Diagnosis. PDW.

\section{Introducción}

El cuadro clínico de apendicitis aguda es la patología quirúrgica de urgencia más frecuente en todo el mundo. Su frecuencia aumenta entre la segunda y la cuarta décadas de la vida. El diagnóstico de apendicitis aguda se establece clínicamente con una tasa de exactitud de hasta el $85 \%$. Las escalas clínicas, como la de Alvarado, poseen una sensibilidad diagnóstica de hasta el $88 \%$ con una especificidad del $75 \%$. Sin embargo, los signos y los síntomas no son específicos y pueden variar dependiendo de cada paciente, lo que hace difícil establecer el diagnóstico con precisión ${ }^{1-3}$.

La tasa de laparotomías/laparoscopías no terapéuticas por probable diagnóstico de apendicitis es del $15 \%$, a pesar del incremento del uso de tecnología en el diagnóstico, como el ultrasonido y la tomografía. Por lo tanto, es necesario contar con nuevas pruebas complementarias para su diagnóstico oportuno. Algunos estudios han investigado la eficacia diagnóstica del ancho de distribución plaquetaria (PDW), un marcador de activación plaquetaria medido en las biometrías hemáticas, como un nuevo parámetro que puede ser utilizado para el diagnóstico de apendicitis aguda ${ }^{3-5}$.

El PDW es un marcador de activación plaquetaria fácilmente medible y específico, debido a que no se incrementa con los cambios temporales del volumen plaquetario (aumento), como lo hacen otros marcadores $^{6}$. El fenómeno de activación plaquetaria provoca cambios morfológicos con incremento de volumen, debido a que las plaquetas obtienen una mayor superficie al cambiar de su forma discoide a una forma esférica con formación de pseudópodos ${ }^{7-9}$. El tamaño de las plaquetas está correlacionado con su actividad y función; las plaquetas más grandes son más activas que las pequeñas, y el PDW es un indicador de cambio en su tamaño, lo que se traduce en un signo de activación plaquetaria. Los valores normales del PDW en los individuos sanos tienen un rango que va del $10 \%$ al $17.9 \%{ }^{10}$.

Existen en la literatura tres estudios que miden la eficacia diagnóstica del PDW en pacientes con apendicitis aguda. En 2013, Aydogan, et al. ${ }^{5}$ fueron los primeros en realizar un estudio retrospectivo de 202 casos, sin grupo control, dividiendo a sus pacientes en dos grupos (apendicitis complicada y no complicada), encontrando valores de PDW significativamente mayores en el grupo de apendicitis complicada y concluyendo que el PDW puede usarse como marcador para la detección temprana del riesgo de perforación apendicular. En 2015, Dinc, et al. ${ }^{3}$ publicaron el primer estudio clínico de casos y controles, comparando 295 casos con dos grupos control, y observaron un incremento significativo del PDW en los pacientes con apendicitis aguda. Reportaron una sensibilidad del $97.1 \%$ y una especificidad de $93 \%$, superior a la sensibilidad de la cifra total de leucocitos y el porcentaje de neutrófilos (73.1\% y $70 \%$, respectivamente), con un valor predictivo positivo del $97.4 \%$ y un valor predictivo negativo del $92.1 \%$. Concluyen que es posible utilizar esta prueba como parte de una batería inicial de herramientas diagnósticas para los pacientes en quienes se sospeche un cuadro de apendicitis, reduciendo costos y pérdida de tiempo en su diagnóstico. En 2015, Fan, et al. ${ }^{4}$ publicaron el segundo estudio de casos y controles, y el primero en estudiar únicamente casos de apendicitis complicada. Observaron un incremento significativo del PDW en este grupo respecto al grupo control de pacientes con apendicitis no complicada, reportando una sensibilidad del $76.3 \%$ y una especificidad del $93.1 \%$, siendo solo superada por la especificidad del porcentaje de neutrófilos, que fue del $96.9 \%$. Sus resultados confirman los de Dinc, et al. ${ }^{3}$, y afirman que el PDW es un parámetro con alta sensibilidad para diagnosticar cuadros de apendicitis complicada ${ }^{4}$.

Estas investigaciones han demostrado un incremento significativo del PDW en los pacientes con apendicitis aguda, sugiriendo a este parámetro como un marcador que puede ser usado para la detección temprana de este cuadro. Sin embargo, es necesario llevar a cabo estudios prospectivos que confirmen estos resultados. A continuación, presentamos un estudio clínico de casos y controles en el que se demuestra la correlación que existe entre el PDW y el diagnóstico de apendicitis aguda.

\section{Método}

Se realizó un estudio de casos y controles con pacientes del Hospital General Dr. Fernando Quiroz Gutiérrez, del Instituto de Seguridad y Servicios Sociales de los Trabajadores del Estado, en el periodo 
comprendido del 1 de marzo al 30 de septiembre de 2016. El estudio fue sometido y aprobado por el comité de ética de dicho hospital. Se incluyeron dos grupos de estudio con muestra calculada empleando los datos demográficos y la incidencia acumulada de los últimos 10 años, conformados por 41 pacientes mayores de 18 años con diagnóstico de apendicitis aguda confirmado por histopatología (grupo I) y 41 sujetos sanos sin comorbilidad, pertenecientes a la población de donadores del banco de sangre del hospital (grupo II). Se excluyeron aquellos pacientes con análisis incompletos y los que no aceptaron el procedimiento quirúrgico. Fueron eliminados del estudio los pacientes en quienes los hallazgos quirúrgicos no correspondieron a apendicitis aguda, así como aquellos con estudio histopatológico no compatible con este diagnóstico.

Se tomaron muestras de sangre venosa de todos los pacientes por personal capacitado, que fueron procesadas en tubos de ensayo con anticoagulante EDTA, utilizando el equipo de biometrías hemáticas del hospital (SYSMEX, marca ROCHE, modelo XN-1000, número de serie 14791), el cual es un equipo automatizado y certificado. Las muestras fueron procesadas en un lapso de tiempo que no excedió las 2 horas.

Las variables analizadas fueron la edad, el sexo, la fase de la apendicitis aguda, el PDW, la cifra total de leucocitos y el porcentaje de neutrófilos. Se utilizó el programa Excel para conformar la base de datos. El análisis estadístico se realizó con el programa IBM ${ }^{\circledR}$ SPSS $^{\circledR}$ Statistics V. 21.

Se midieron las modificaciones del PDW, la cifra total de leucocitos y el porcentaje de neutrófilos en los pacientes con apendicitis aguda y en el grupo control, así como las diferencias del PDW entre hombres y mujeres en ambos grupos. Se utilizó la prueba estadística $U$ de Mann-Whitney, debido a que las variables estudiadas no tuvieron una distribución normal, y se realizó una regresión lineal para determinar la relación del PDW, la cifra total de leucocitos y el porcentaje de neutrófilos con las fases de la apendicitis aguda. Finalmente, se realizó una comparación de los datos encontrados con los reportados en la literatura médica internacional.

\section{Resultados}

La edad media fue de $37.27 \pm 15.6$ años; hubo una mayor cantidad de pacientes en edades inferiores a los 50 años (Fig. 1). En cuanto a la distribución por sexo, se observó una mayor frecuencia del sexo femenino, con un $56 \%$ del total en el grupo de casos (Fig. 2). En cuanto a la fase de la apendicitis en que se midieron

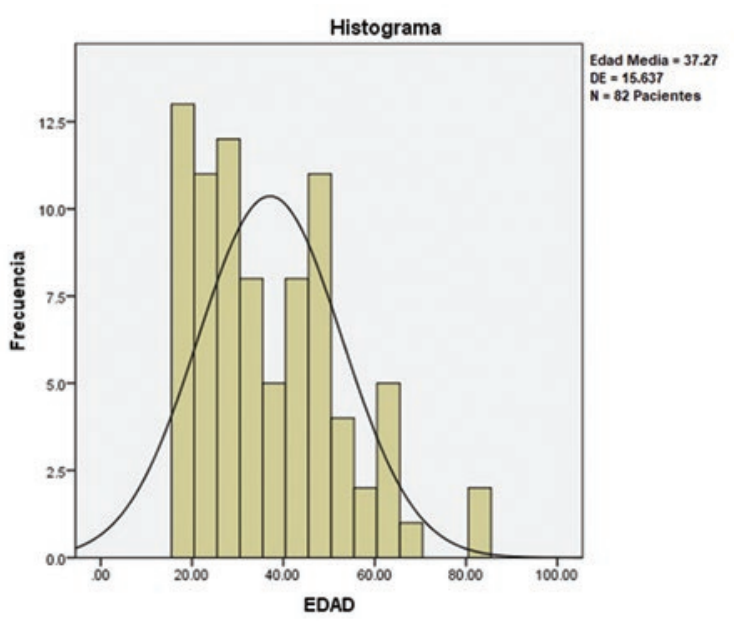

Figura 1. Distribución de la edad de los pacientes estudiados. El eje y representa la frecuencia de presentación y el eje x representa la edad. $D E$ : desviación estándar.

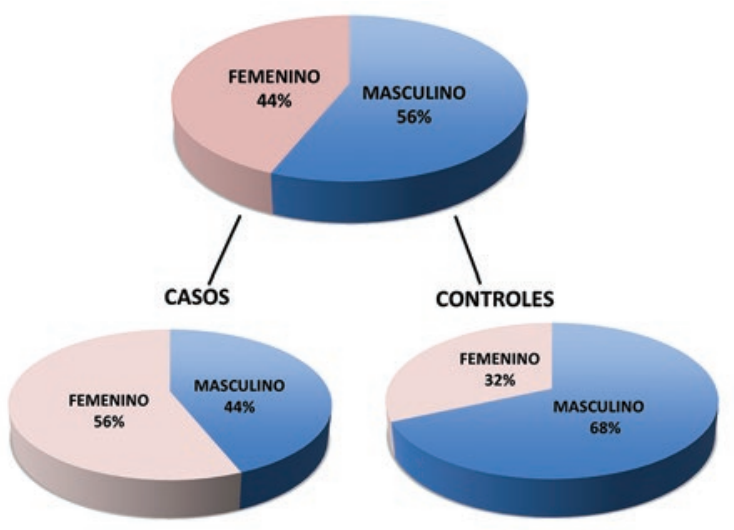

Figura 2. Distribución por sexo de los pacientes estudiados.

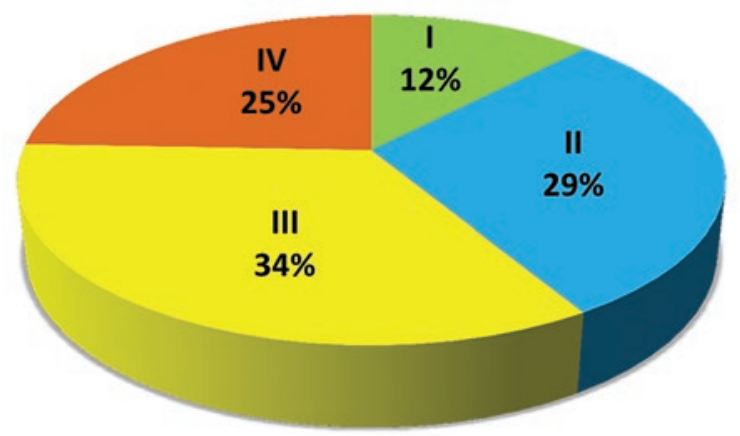

Figura 3. Distribución de la frecuencia de las fases de la apendicitis en que se presentaron los casos estudiados.

las variables, la mayor cantidad de casos se presentaron en fase III, con un $34 \%$ del total (Fig. 3). La cifra de leucocitos y el porcentaje de neutrófilos mostraron 
Tabla 1. Distribución de los valores de tendencia central y dispersión de las variables analizadas en la biometría hemática

\begin{tabular}{lccccc}
\hline Variable & \multicolumn{2}{c}{ Casos } & & \multicolumn{2}{c}{ Controles } \\
\cline { 2 - 3 } \cline { 5 - 6 } & \begin{tabular}{c} 
Media \\
\cline { 5 - 6 }
\end{tabular} & $\begin{array}{c}\text { Desviación } \\
\text { estándar }\end{array}$ & & Media & $\begin{array}{c}\text { Desviación } \\
\text { estándar }\end{array}$ \\
\hline $\begin{array}{l}\text { Ancho de distribución } \\
\text { plaquetaria }\end{array}$ & 13.54 & 3.1 & 13.53 & 1.06 \\
Cifra total de leucocitos & 13.35 & 4.42 & 7.07 & 1.61 \\
Porcentaje de neutrófilos & 79.97 & 8.34 & 58.79 & 9.33 \\
\hline
\end{tabular}

Tabla 2. Prueba de hipótesis para las variables analizadas en la biometría hemática

\begin{tabular}{|c|c|c|c|}
\hline Variable & Valor Mann-Whitney & Wilcoxon & $\mathrm{p}$ \\
\hline $\begin{array}{l}\text { Ancho de distribución } \\
\text { plaquetaria }\end{array}$ & 634.5 & $1,495.5$ & 0.54 \\
\hline Cifra total de leucocitos & 125 & 986 & 0.0001 \\
\hline $\begin{array}{l}\text { Porcentaje de } \\
\text { neutrófilos }\end{array}$ & 109.5 & 970.5 & 0.0001 \\
\hline
\end{tabular}

Tabla 3. Regresión lineal de la fase de apendicitis con el ancho de distribución plaquetaria, la cifra total de leucocitos y el porcentaje de neutrófilos

\begin{tabular}{lcc}
\hline Variables en el modelo & B & p \\
\hline Cifra total de leucocitos & 0.754 & 0.0001 \\
Porcentaje de neutrófilos & 0.735 & 0.0001 \\
Ancho de distribución plaquetaria & -0.018 & 0.95 \\
\hline
\end{tabular}

Tabla 4. Prueba de hipótesis para ancho de distribución plaquetaria y sexo en pacientes con apendicitis

\begin{tabular}{lcc}
\hline Variable & Mann-Whitney & $\mathbf{p}$ \\
\hline Sexo & 145 & 0.101 \\
\hline
\end{tabular}

diferencias estadísticamente significativas en comparación con el grupo control (Tablas 1 y 2). Se observó que no había una asociación entre los valores del PDW y la fase de la apendicitis aguda, pero sí hubo una asociación directamente proporcional para la cifra total de leucocitos y el porcentaje de neutrófilos con las fases de la apendicitis (Tabla 3). En cuanto a la asociación del PDW con el sexo de los pacientes, tampoco se hallaron diferencias (Tabla 4).

\section{Discusión}

La apendicitis aguda es la causa más frecuente de abdomen agudo en todos los grupos de edad. Realizar el diagnóstico de apendicitis aguda de manera rápida y precisa es importante debido a que el alargamiento del tiempo desde el inicio de los síntomas hasta la entrada del paciente al quirófano incrementa el riesgo de complicaciones, como perforación apendicular, peritonitis, sepsis abdominal e incluso la muerte. Por ello, las pruebas de laboratorio con altas sensibilidad y especificidad resultan estudios cruciales para establecer diagnósticos diferenciales confiables ${ }^{1-3}$.

La cuenta leucocitaria es uno de los estudios más usados, por ser el primer parámetro en elevarse durante un proceso apendicular; en el presente estudio, los valores de corte fueron de $13.35 \times 10^{\%} / /$, concordantes con los reportados en la literatura'11. De igual forma, numerosos estudios han evaluado la eficacia del recuento diferencial de neutrófilos para el diagnóstico de pacientes con apendicitis; en este estudio, el valor de corte fue del $79.97 \%$, similar a lo informado internacionalmente.

Existen en la literatura tres estudios que correlacionan el PDW con la apendicitis aguda: el de Aydogan, et al. ${ }^{5}$ en 2013, el de Dinc, et al. ${ }^{3}$ en 2015 y el de Fan, et al. ${ }^{4}$ también en 2015. Estas investigaciones demostraron un incremento significativo del PDW, sugiriéndolo como un marcador que puede ser usado para la detección temprana de pacientes con apendicitis aguda.

En el presente estudio prospectivo, la muestra fue calculada y nos permite determinar que las diferencias no encontradas no se deben a un problema de tamaño de muestra, sino a que no existen diferencias en los valores observados del PDW. La edad de los pacientes traduce que la patología apendicular aguda se presenta mayoritariamente en adultos jóvenes, lo que permite pensar que posibles padecimientos concomitantes no sesgaron los resultados aquí presentados.

Solo fue posible determinar diferencias en la cifra total de leucocitos y en el porcentaje de neutrófilos, con valores de $p<0.05$, así como una asociación estadísticamente significativa del incremento de estas dos variables con las fases de la apendicitis; situación esperada ya que el grupo de comparación es un grupo sano, pero el PDW no mostró diferencias estadísticamente significativas.

El diagnóstico de apendicitis aguda debe combinar siempre la clínica con el laboratorio y los estudios de imagen, y a pesar de que un biomarcador puede ser un parámetro de evaluación importante, siempre tiene que ser correlacionado con los síntomas (migración del dolor, 
vómito, etc.), los signos en la exploración física (dolor en la fosa iliaca derecha, signo de McBurney o sucedáneos), las pruebas de laboratorio (leucocitosis, aumento del porcentaje de neutrófilos) y los estudios de gabinete (radiografía simple de abdomen, ultrasonido, tomografía).

Los objetivos de esta investigación fueron observar las modificaciones del PDW en los pacientes con apendicitis aguda, su relación con la fase de la apendicitis y las diferencias entre hombres y mujeres con este padecimiento. Estos objetivos se cumplieron al observar que no hubo modificaciones en el PDW en ninguno de los parámetros evaluados.

\section{Conclusiones}

No hay diferencias entre los valores del PDW de los pacientes con apendicitis aguda en comparación con sujetos sanos, ni relación del PDW con las fases de la apendicitis, por lo que consideramos, según los resultados obtenidos en el presente estudio, que el PDW no es un marcador útil para el diagnóstico de apendicitis aguda. Sin embargo, es necesario realizar nuevos estudios prospectivos y multicéntricos que confirmen estos resultados.

\section{Agradecimientos}

Al personal del banco de sangre del Hospital General Dr. Fernando Quiroz Gutiérrez por la disponibilidad y las facilidades prestadas para la realización del presente estudio. A los doctores Carlos Alberto Pantoja Meléndez, Jazmin Escobar Juárez y Axel Edmundo Guzmán Cedillo, por su apoyo para el análisis estadístico y metodológico.

\section{Conflicto de intereses}

Los autores declaran no tener ningún conflicto de intereses.

\section{Financiamiento}

Los autores declaran no haber recibido ningún patrocinio para llevar a cabo este artículo.

\section{Responsabilidades éticas}

Protección de personas y animales. Los autores declaran que para esta investigación no se han realizado experimentos en seres humanos ni en animales.

Confidencialidad de los datos. Los autores declaran que han seguido los protocolos de su centro de trabajo sobre la publicación de datos de pacientes.

Derecho a la privacidad y consentimiento informado. Los autores declaran que en este artículo no aparecen datos de pacientes.

\section{Bibliografía}

1. Paulson EK, Kalady MF, Pappas TN. Clinical practice. Suspected appendicitis. N Engl J Med. 2003;348:236-42.

2. Rebollar González RC, García Álvarez J, Trejo Téllez R. Apendicitis aguda: revisión de la literatura. Rev Hosp Jua Mex. 2009;76:210-6.

3. Dinc B, Oskay A, Dinc SE, Bas B, Tekin S. New parameter in diagnosis of acute appendicitis: platelet distribution width. World J Gastroenterol. 2015;21:1821-6.

4. Fan Z, Pan J, Zhang Y, Wang Z, Zhu M, Yang B, et al. Mean platelet volume and platelet distribution width as markers in the diagnosis of acute gangrenous appendicitis. Dis Markers. 2015;2015:542013.

5. Aydogan A, Akkucuk S, Arica S, Motor S, Karakus A, Ozkan OV, et al. The analysis of mean platelet volume and platelet distribution width levels in appendicitis. Indian J Surg. 2015;77(Suppl 2):495-500.

6. Vagdatli E, Gounari E, Lazaridou E, Katsibourlia E, Tsikopoulou F, Labrianou I. Platelet distribution width: a simple, practical and specific marker of activation of coagulation. Hippokratia. 2010;14:28-32.

7. Shah AR, Chaudhari SN, Shah MH. Role of platelet parameters in diagnosing various clinical conditions. Nat J Med Res. 2013;3:162-5.

8. Sachdev R, Tiwari AK, Goel S, Raina V, Sethi M. Establishing biological reference intervals for novel platelet parameters (immature platelet fraction, high immature platelet fraction, platelet distribution width, platelet large cell ratio, platelet-X, plateletcrit, and platelet distribution width) and their correlations among each other. Indian J Pathol Microbiol. 2014;57:231-5.

9. Gasparyan AY, Ayvazyan L, Mikhailidis DP, Kitas GD. Mean platelet volume: a link between thrombosis and inflamation? Curr Pharm Des. 2011:17:47-58.

10. Farias MG, Schunck EG, Dal Bó S, Martins de Castro S. Definition of reference ranges for the platelet distribution width (PDW): a local need. Clin Chem Lab Med. 2010;48:255-7.

11. Al-Gaithy ZK. Clinical value of total white blood cells and neutrophil counts in patients with suspected appendicitis: retrospective study. World J Emerg Surg. 2012;7:32. 